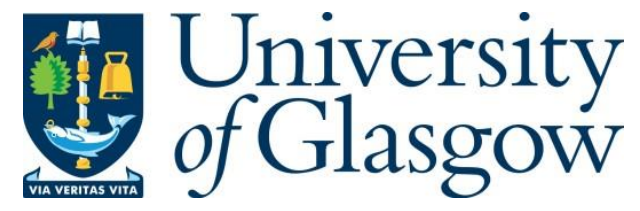

Lee, J., Kim, S. and Shin, M. (2017) A theoretical model for predicting Schottky-barrier height of the nanostructured silicide-silicon junction. Applied Physics Letters, 110(23), 233110. (doi:10.1063/1.4985013)

This is the author's final accepted version.

There may be differences between this version and the published version. You are advised to consult the publisher's version if you wish to cite from it.

http://eprints.gla.ac.uk/143024/

Deposited on: 26 June 2017

Enlighten - Research publications by members of the University of Glasgow http://eprints.gla.ac.uk33640 


\title{
A theoretical model for predicting Schottky-barrier height of the nanostructured silicide-silicon junction
}

\author{
Jaehyun Lee, ${ }^{1, \text { a) }}$ Seungchul Kim, ${ }^{2, \text { b) }}$ and Mincheol Shin*1, c) \\ ${ }^{1)}$ School of Electrical Engineering, KAIST, 291 Daehak-ro, Yuseong-gu, Daejeon 34141, \\ Rep. of Korea \\ ${ }^{2)}$ Computational Science Research Center, KIST, 5 Hwarang-ro, 14-gil, Seongbuk-gu, Seoul 02792, \\ Rep. of Korea
}

(Dated: 16 May 2017)

In this work, we have performed the first-principles calculations to investigate the Schottky-barrier height $(\mathrm{SBH})$ of the various nanostructured silicide-silicon junctions. As for the silicides, PtSi, NiSi, TiSi 2 , and $\mathrm{YSi}_{2}$ have been used. We find that $E_{\mathrm{FiF}}=E_{\mathrm{Fi}}-E_{\mathrm{F}}$, where $E_{\mathrm{Fi}}$ and $E_{\mathrm{F}}$ are the intrinsic Fermi level of the semiconductor part and the Fermi level of the junction, respectively, is unchanged by nanostructuring. From this finding, we suggest a model, a symmetric increase of SBH (SI) model, to properly predict SBHs of nanostructured silicide-silicon junctions. We also suggest two measurable quantities for experimental validation of our model. The effect of our SI model applied to nanostructures such as nanowire and ultrathin-body is compared with that of the widely used previous SBH model.

Schottky-barrier (SB) metal-oxide-semiconductor field-effect-transistors (MOSFETs), which have metallic source/drain (S/D) instead of heavily doped semiconductor, have been regarded as one of the most promising candidates for the future electronic devices ${ }^{1-6}$. As the size of devices has continued to shrink to the nanoscale, high parasitic resistance and capacitance caused by the shallow junction have become an inevitable problem. The metallic S/D of nano-scaled SB-MOSFETs is of benefit to solve this problem. Moreover, these devices have other merits such as low-thermal budget and sharp interfaces between the metal and semiconductor.

The main obstacle in developing SB-MOSFETs is their low ON-state current $\left(I_{\mathrm{ON}}\right)^{7-10}$. This is because, unlike the conventional MOSFETs, electric current flows through quantum tunneling effect at the junction such that $I_{\mathrm{ON}}$ decreases exponentially to the height of SB $\left(\mathrm{SBH}, \phi_{\mathrm{b}}\right)$. To enhance $I_{\mathrm{ON}}$, mainly there are two approaches; one is to replace the channel material with the one that has low effective masses, and the other is to lower the SBH at the interface between the metal and the semiconductor. According to the previous theoretical works ${ }^{8,10}$, the $\mathrm{SBH}$ reduction seems more effective than the channel material engineering for enhancing $I_{\mathrm{ON}}$. In particular, Guo et al. ${ }^{8}$ showed that $I_{\mathrm{ON}}$ of ultra-thinbody (UTB) SB-MOSFETs becomes comparable to that of conventional UTB MOSFETs when SBH becomes negative.

Not much progress has been made in modeling of $\mathrm{SBH}$ of the nanostructure during the last few decades. Almost all theoretical models on SB describe SBH of bulk junction $\left(\phi_{\mathrm{b}}^{\mathrm{Bulk}}\right)$ with empirical parameters rather than predicts SBH from first principles. Indeed, understanding on $\mathrm{SBH}$ of the nanostructure is still in an early stage.

\footnotetext{
a) jaehyun.lee@kaist.ac.kr

b) sckim@kist.re.kr

c) mshin@kaist.ac.k
}

It is well-known that SBH of the nanostructured junction $\left(\phi_{\mathrm{b}}^{\mathrm{Nano}}\right)$ is larger than $\phi_{\mathrm{b}}^{\mathrm{Bulk}}$ due to the increment of the band gap $\left(E_{\mathrm{g}}\right)$ by the quantum confinement effect ${ }^{11,12}$. In the previous studies ${ }^{8-10}, \phi_{\mathrm{b}}^{\mathrm{Nano}}$ have been defined by $\phi_{\mathrm{bp}(\mathrm{n})}^{\mathrm{Nano}}=\phi_{\mathrm{bp}(\mathrm{n})}^{\mathrm{Bulk}}+E_{\mathrm{QV}(\mathrm{C})}$, where $\phi_{\mathrm{bp}(\mathrm{n})}$ is $\mathrm{SBH}$ for holes (electrons), and $E_{\mathrm{QV}(\mathrm{C})}$ is the quantization energy of valence band (conduction band). Because $E_{\mathrm{QV}} \neq E_{\mathrm{QC}}$ in general, we call this model "asymmetric increase of $\mathrm{SBH}$ (AI) model" hereafter. Although the AI model for the nanostructures provides intuitive and empirical understanding to some observations, it has never been validated experimentally nor verified by a rigorous theory. Namely, the study on SBH of the nanostructured junction is still lacking. ${ }^{13}$

In recent years, a number of studies have attempted to calculate $\mathrm{SBH}$ by using the density functional theory $(\mathrm{DFT})^{11,12,14-24}$. Unlike the classical models such as Schottky-Mott (SM) rule 25,26 and metal induced gap states (MIGS) model ${ }^{27,28}$, the DFT method has the merits of considering and examining interatomic effects between semiconductor and metal atoms. While most of the DFT studies on SBH focused on bulk junction, E. Montes et $a l .{ }^{11}$ and U. Landman et al. ${ }^{12}$ have demonstrated a DFT calculation for a nanostructured junction and showed that $\phi_{\mathrm{b}}^{\mathrm{Nano}}$ is larger than $\phi_{\mathrm{b}}^{\mathrm{Bulk}}$ as expected. However, they did not analyze the relationship between $\phi_{\mathrm{b}}^{\mathrm{Nano}}$ and $\phi_{\mathrm{b}}^{\mathrm{Bulk}}$.

In this work, we perform the DFT calculations for various nanostructured silicide-silicon junctions to investigate their SBHs. As for the silicides, PtSi, NiSi, $\mathrm{TiSi}_{2}$ and $\mathrm{YSi}_{2}$ are used. Due to the well-known band gap underestimation problem of DFT calculations, we focus on the change of SBHs from the bulk to the nanostructured junctions rather than to find absolute values of SBH. The main purpose of this investigation is to explore the relationship between $\phi_{\mathrm{b}}^{\mathrm{Nano}}$ and $\phi_{\mathrm{b}}^{\mathrm{Bulk}}$, and to provide a model to predict SBH of nanostructured silicide-silicon junction.

For this purpose, we used the SIESTA package ${ }^{29,30}$. 


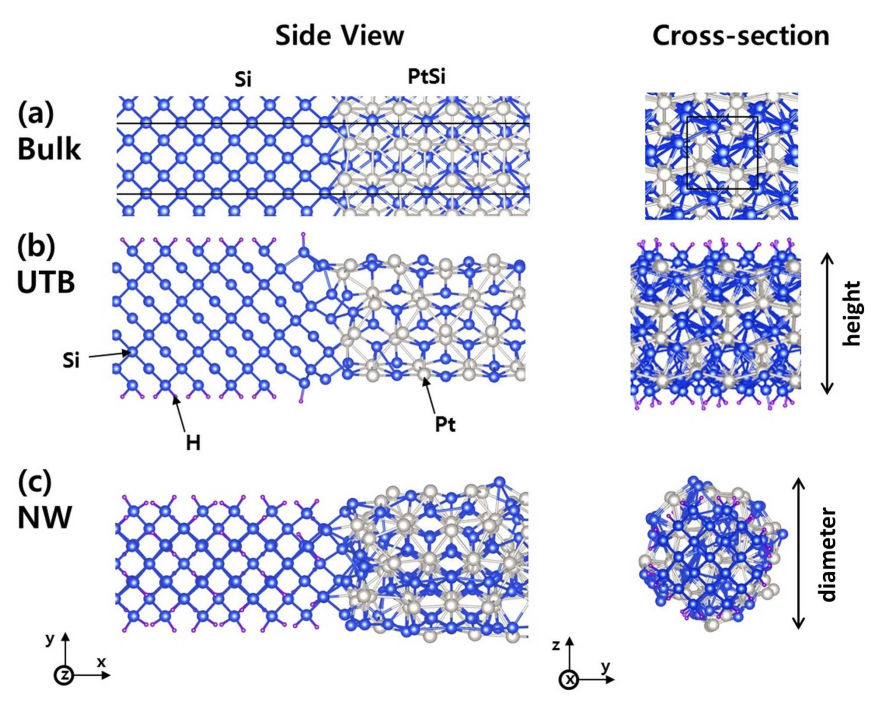

FIG. 1. Side view and cross-section of relaxed atomic structures of (a) PtSi-Si bulk, (b) PtSi-Si UTB $(h=1.1 \mathrm{~nm})$, and (c) PtSi-Si nanowire (NW) $(d=1.0 \mathrm{~nm})$, respectively. The solid black lines indicate the unit cell of junctions. To model the junction structure, we use the supercell approximation along the x-axis. Blue, gray, and violet balls symbolize the $\mathrm{Si}, \mathrm{Pt}$, and $\mathrm{H}$ atoms, respectively. The nanostructures such as (b) UTB and (c) NW are separated by the vacuum region of about $15 \AA$.

The generalized gradient approximation (GGA) is applied for the exchange-correlation energy functional, as proposed by Perdew, Burke, and Ernzerhof $(\mathrm{PBE})^{31}$. Troullier-Martins type of normconserving pseudopotential ${ }^{32}$ with scalar-relativistic correction, double- $\zeta$ singly-polarized (DZP) pseudo atomic orbital basis set, and $100 R y$ of cutoff energy for electron density are used. The atoms are relaxed until the maximum force on any of the atoms becomes less than 0.04 $\mathrm{eV} / \AA$.

The silicide-silicon junctions are modeled using the supercell approximation, which consists of large silicon and silicide parts in the unit cell (see Fig. 1). It is assumed that PtSi and NiSi have orthorhombic crystal structures, and $\mathrm{TiSi}_{2}$ and $\mathrm{YSi}_{2}$ have base-centered orthorhombic (C49 phase) and hexagonal $\mathrm{AlB}_{2}$ crystal structures, respectively. For modeling the bulk junctions, we assume that the lattice constant of each silicide is adjusted to match that of the bulk Si. The averages of the lattice mismatches along $y$ - and $z$-axis are $8,1,-7$ and $6 \%$ for $\mathrm{PtSi}, \mathrm{NiSi}, \mathrm{TiSi}_{2}$, and $\mathrm{YSi}_{2}$, respectively. The lattice constant along $x$-axis is chosen when the total energy is minimized. In the case of the UTB structure, $\mathrm{Si}$ orientation may be differently applied for different silicide to minimize the lattice mismatch; for example, we use $\mathrm{Si}(100) /[001]$ for $\mathrm{PtSi}$ and $\mathrm{NiSi}$, and $\mathrm{Si}(100) /[011]$ for $\mathrm{YSi}_{2}$ and $\mathrm{TiSi}_{2}$, respectively, where $(l m n) /[l m n]$ denotes the transport/confinement directions, respectively. Note that one should carefully choose the confinement direction of the silicide not to change its work-function compared with that of the bulk. Dangling bonds on the surface of Si part were passivated with hydrogen atoms.

When considering the relationship between SBH and silicide work-function $\left(\Phi_{\mathrm{M}_{\mathrm{m}} \mathrm{Si}_{\mathrm{n}}}\right)$ as shown in Figs. 2 and 3 , it is no wonder that variation in the work-function produces large change in SBH. Therefore, we shall not comment on a change in the work-function caused by the structural variation of the silicide.

We obtain the theoretical SBH for hole $\left(\phi_{\mathrm{bp}}\right)$ by finding the difference between the Fermi level $\left(E_{\mathrm{F}}\right)$ and the valence band maximum energy $\left(E_{\mathrm{VBM}}\right)$ of silicon atoms farther away from the decay length of the in-gap state at the junction. $\phi_{\mathrm{bp}}$ is defined by the following equations;

$$
\phi_{\mathrm{bp}}=E_{\mathrm{F}}-E_{\mathrm{VBM}}=\frac{1}{2} E_{\mathrm{g}}-\left(E_{\mathrm{Fi}}^{\mathrm{Si}}-E_{\mathrm{F}}\right)
$$

where $E_{\mathrm{Fi}}^{\mathrm{Si}}$ is the intrinsic Fermi level of silicon (i.e. $\left.\frac{1}{2}\left(E_{\mathrm{CBM}}+E_{\mathrm{VBM}}\right)\right)$, and $E_{\mathrm{CBM}}$ is the conduction band minimum energy. $E_{\mathrm{VBM}}$ and $E_{\mathrm{CBM}}$ are obtained from the projected density of states. Note that the band gap (i.e. $\left.E_{\mathrm{CBM}}-E_{\mathrm{VBM}}\right)$ is $0.55 \mathrm{eV}$ (49\% of experimental value) in our calculation.

The dependence of experimental ${ }^{33-44}$ and theoretical $\phi_{\mathrm{bp}}^{\text {Bulk }}$ on $\Phi_{\mathrm{M}_{\mathrm{m}} \mathrm{Si}_{\mathrm{n}}}$ is shown in Fig. 2. Experimental $\Phi_{\mathrm{M}_{\mathrm{m}} \mathrm{Si}_{\mathrm{n}}}$ is estimated in the Miedema style as a geometrical averaged work function of the metal and silicon, $\Phi_{\mathrm{M}_{\mathrm{m}} \mathrm{Si}_{\mathrm{n}}}=\left(\Phi_{\mathrm{M}}^{\mathrm{m}} \Phi_{\mathrm{Si}}^{\mathrm{n}}\right)^{1 /(\mathrm{m}+\mathrm{n})}$, while theoretical $\Phi_{\mathrm{M}_{\mathrm{m}} \mathrm{Si}_{\mathrm{n}}}$ is obtained from the DFT calculations by taking the difference between the vacuum level $\left(E_{\mathrm{vac}}\right)$ and the Fermi level of silicide $\left(E_{\mathrm{F}}^{\mathrm{M}_{\mathrm{m}} \mathrm{Si}_{\mathrm{n}}}\right)$ in the slab structure. As Fig. 2 shows, the theoretical $\phi_{\text {bp }}$ linearly decreases as $\Phi_{\mathrm{M}_{\mathrm{m}} \mathrm{Si}_{\mathrm{n}}}$ increases in the same manner as the experimental results. Moreover, the Fermi level pinning effect is shown in both the experimental and theoretical results; the slope $(S)$ between $\phi_{\mathrm{bp}}^{\text {Bulk }}$ and $\Phi_{\mathrm{M}_{\mathrm{m}} \mathrm{Si}_{\mathrm{n}}}$ is less than that predicted by the SM rule. Note that the calculated $S$ parameter of the bulk $(0.22)$ is comparable to the experimental value of 0.36 in spite of $51 \%$ of band gap error that GGA calculation has. We can therefore remark that the DFT approach is appropriate to describe the change of SBH.

Fig. 3 presents the theoretical $\phi_{\mathrm{bp}}$ of the bulk and nanowires (NWs) with a diameter $(d)$ of 1.0 and $1.5 \mathrm{~nm}$, respectively. Due to the confinement effects, the smaller structure, the higher SBH shows. We pay a particular attention to the observation that the $S$ parameters of the bulk and NWs are quite close to each other ( 0.22 for bulk, 0.18 for $1 \mathrm{~nm} \mathrm{NWs}$, and 0.22 for $1.5 \mathrm{~nm} \mathrm{NWs}$ ) although there is a significant difference in their atomic structures as shown in Fig. 1. This insensitivity of $S$ on the size of the junction can be interpreted as insensitivity of SBH on the size change of each silicide because, for a given silicide, it was found that work functions of all nanostructures are the same as that of the bulk. To confirm this, we redraw SBH as a function of the band gap of each structure.

SBHs for hole of PtSi-Si and $\mathrm{YSi}_{2}-\mathrm{Si}$ junctions almost perfectly change linearly with respect to the band gap of the nanostructured $\mathrm{Si}$, as Fig. 4 shows. The slope 


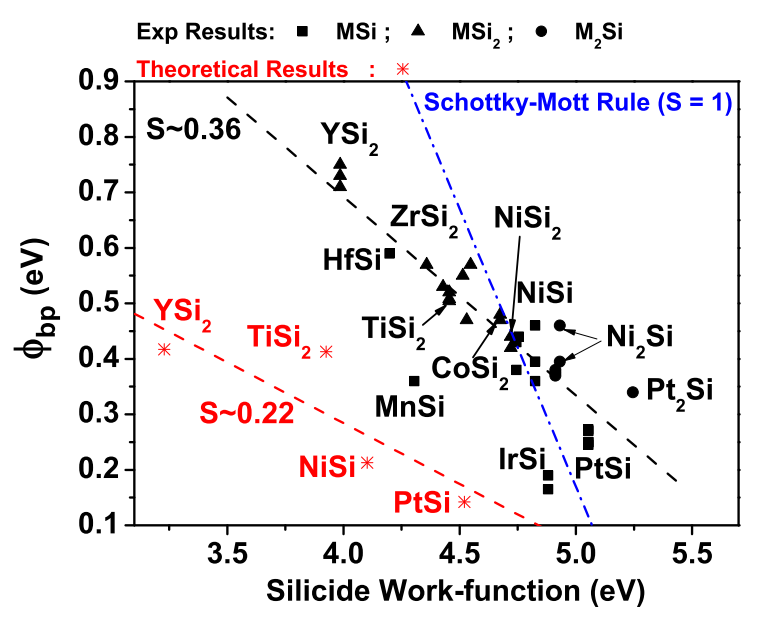

FIG. 2. Experimental ${ }^{33-44}$ and theoretical SBHs for hole are plotted against the work-function of silicides. $M$ in the legend indicates the metal atom. We assume that $\phi_{\mathrm{bp}}+\phi_{\mathrm{bn}}=E_{\mathrm{g}}$.

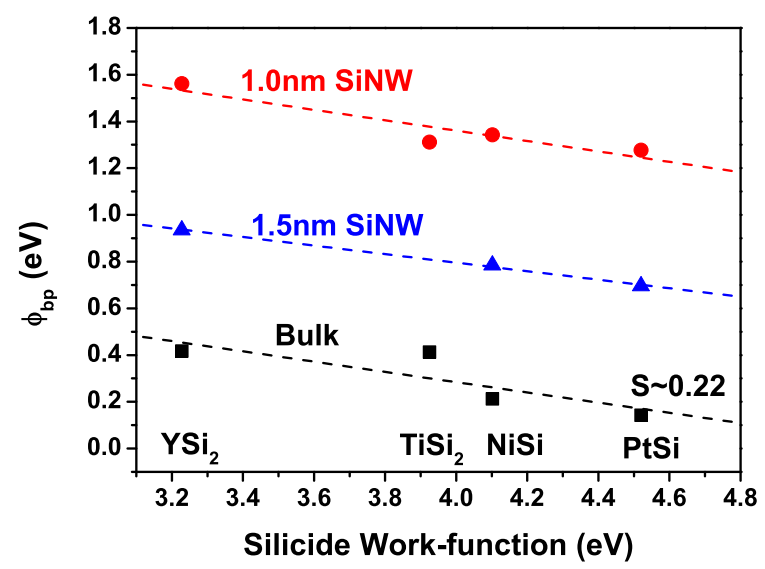

FIG. 3. Theoretical SBHs for hole of the bulk and NW $(d=$ 1.0 and $1.5 \mathrm{~nm}$ ) junctions are plotted as a function of calculated work-function of the bulk silicides.

for both $\mathrm{PtSi}$ and $\mathrm{YSi}_{2}$ are 0.5 , which leads to the most important message of this study that $E_{\mathrm{FiF}}=E_{\mathrm{Fi}}^{\mathrm{Si}}-E_{\mathrm{F}}$ is constant regardless of the size of nanostructures. $E_{\mathrm{FiF}}$ depends only on the kind of silicides, therefore SBH of the nanostructured junction is predictable if we know $E_{\mathrm{FiF}}$ and band gap of the nanostructured Si. The constant $E_{\mathrm{FiF}}$ is observed in both the $p$-type $(\mathrm{PtSi})$ and $n$-type $\left(\mathrm{YSi}_{2}\right)$ silicides.

From the above observation, we suggest a model to predict $\phi_{\mathrm{bp}}^{\mathrm{Nano}}$ from $\phi_{\mathrm{bp}}^{\mathrm{Bulk}}$;

$$
\begin{aligned}
\phi_{\mathrm{bp}}^{\text {Nano }} & =\frac{1}{2} E_{\mathrm{g}}^{\text {Nano }}-E_{\mathrm{FiF}} \\
& =\phi_{\mathrm{bp}}^{\text {Bulk }}+\frac{1}{2}\left(E_{\mathrm{g}}^{\text {Nano }}-E_{\mathrm{g}}^{\text {Bulk }}\right)
\end{aligned}
$$

where $E_{\mathrm{g}}^{\text {Nano }}$ and $E_{\mathrm{g}}^{\text {Bulk }}$ are band gaps of the nanostructured and bulk semiconductors, respectively. We call this

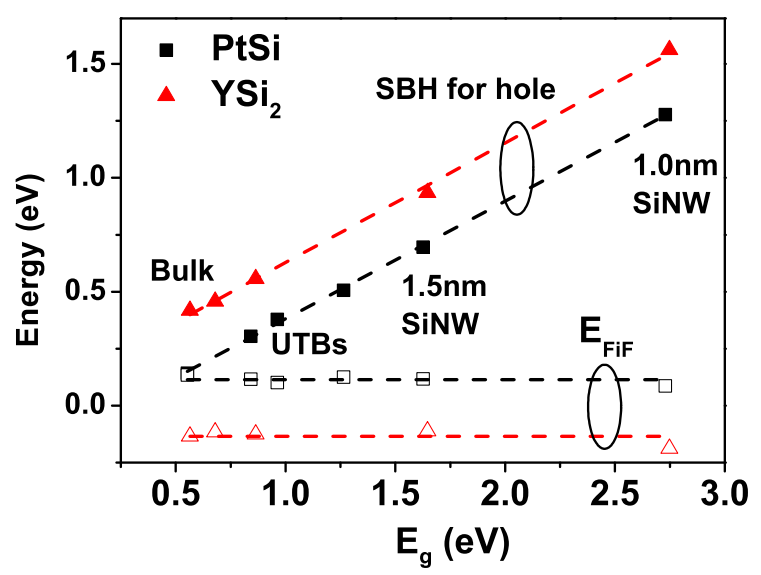

FIG. 4. The dependence of the SBH for hole and $E_{\mathrm{FiF}}=$ $E_{\mathrm{Fi}}^{\mathrm{Si}}-E_{\mathrm{F}}$ on the band gap is shown. The band gap of the bulk $\mathrm{Si}$ is $0.55 \mathrm{eV}$ in GGA-PBE calculations.

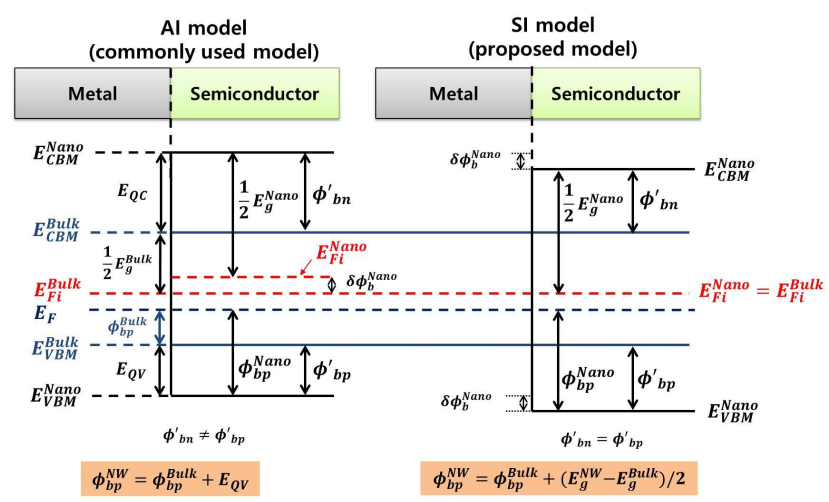

FIG. 5. The schematic band diagrams of the AI and SI models. It is assumed that $E_{\mathrm{F}}$ is fixed.

model "symmetric increase of SBH (SI) model" hereafter. This is because, unlike the commonly used AI model, $\phi_{\mathrm{bp}}^{\prime}=\phi_{\mathrm{bp}}^{\mathrm{Nano}}-\phi_{\mathrm{bp}}^{\mathrm{Bulk}}$ and $\phi_{\mathrm{bn}}^{\prime}=\phi_{\mathrm{bn}}^{\mathrm{Nano}}-\phi_{\mathrm{bn}}^{\mathrm{Bulk}}$ are exactly the same in this model (see Fig. 5).

Fig. 5 illustrates the schematic band diagrams of the bulk and nanostructured junctions to compare the AI model with the SI model. In both models, the band gap increases by nanostructuring have the same value and are determined by the well-known quantum confinement effect. When a junction is formed in the nanostructure, the main difference between the two models is that $E_{\mathrm{Fi}}$ 's of the nanostructured junction and bulk junction are the same (i.e. $E_{\mathrm{Fi}}^{\mathrm{Nano}}=E_{\mathrm{Fi}}^{\mathrm{Bulk}}$ ) in the SI model while they are different in the AI model. This difference results in the difference in the predicted value for $\phi_{\mathrm{b}}^{\mathrm{Nano}}$ between the two models;

$$
\delta \phi_{\mathrm{b}}^{\mathrm{Nano}}=\left|\phi_{\mathrm{bp}(\mathrm{n})}^{\mathrm{Nano}, \mathrm{AI}}-\phi_{\mathrm{bp}(\mathrm{n})}^{\mathrm{Nano}, \mathrm{SI}}\right|=\frac{1}{2}\left|E_{\mathrm{QC}}-E_{\mathrm{QV}}\right| \cdot(4)
$$

This disparity raises questions as to which model is more relevant to nanostructured junction. Unfortunately we 
were not able to find the experimental results proper to test the theoretical models, but we propose a method to test them as will be mentioned shortly.

To verify the plausibility of our SI model, we compare it with other empirical models, the MIGS model and the $\mathrm{SM}$ rule. We find that both the models are indeed commensurate well with the SI model, whereas there is no such relevance with regard to the AI model. Firstly, the non-ideal $\phi_{\mathrm{bp}}$ by the MIGS model is given by

$$
\phi_{\mathrm{bp}}=\frac{1}{2} E_{\mathrm{g}}-\left\{S\left(E_{\mathrm{CNL}}-E_{\mathrm{F}}^{\mathrm{M}_{\mathrm{m}} \mathrm{Si}_{\mathrm{n}}}\right)-E_{\mathrm{CNL}}+E_{\mathrm{Fi}}^{\mathrm{Si}}\right\}
$$

where $E_{\mathrm{CNL}}$ is the charge neutrality level (CNL) of Si. By the Tersoff's theory ${ }^{45}$, it is clear that $\Phi_{\mathrm{CNL}}=$ $E_{\mathrm{vac}}-E_{\mathrm{CNL}}$ is independent on the kind of silicide. This agrees with our DFT results in Fig. 1; the calculated $\Phi_{\mathrm{CNL}}$ 's show quite similar values of $4.112,4.065$, and $4.021 \mathrm{eV}$ for the bulk, NW $(d=1.0 \mathrm{~nm})$, and NW $(d$ $=1.5 \mathrm{~nm}$ ), respectively. If the $S$ parameter and $E_{\mathrm{CNL}}$ are independent of the kind of silicide and junction structure, $\left\{S\left(E_{\mathrm{CNL}}-E_{\mathrm{F}}^{\mathrm{M}_{\mathrm{m}} \mathrm{Si}_{\mathrm{n}}}\right)-E_{\mathrm{CNL}}\right\}$ can be replaced by $-E_{\mathrm{F}}$, which corresponds to the Fermi level pinning effect. Then, Eq. (5) can be rewritten as Eq. (2). In the case of the SM rule $(S=1), E_{\mathrm{F}}$ becomes equal in $E_{\mathrm{M}_{\mathrm{m}} \mathrm{Si}_{\mathrm{n}}}$ and again Eq. (2) is applicable. The connection between the SI model and the well-known classical models is by no means a coincidence.

Any proposed theoretical model should be validated against experimental data. However, we were not able to find enough set of experimental data for the validation. Instead, we suggest the following two physical quantities that can be measured in experiments and can be used to judge whether the SI model is more relevant to nanostructured junctions than the AI model. They are $\frac{d \phi_{\mathrm{bp}(\mathrm{n})}}{d E_{\mathrm{g}}}$ and $\frac{d\left(\log _{10} J_{0}\right)}{d E_{\mathrm{g}}}$, where $J_{0}$ is the saturation current density. Fig. 6 shows the predicted results from the SI and AI models for $\mathrm{Si}$ and InAs, where the data were obtained by numerical calculations by the $s p^{3} d^{5} s^{*}$ empirical tightbinding (TB) method.

In Fig. 6, the SI model gives $\frac{d \phi_{\mathrm{bp}(\mathrm{n})}}{d E_{\mathrm{g}}}=0.5$ for both $\mathrm{Si}$ and InAs, which is a direct consequence from the assumption of the SI model (see Eq. (3)). On the other band, the AI model gives $\frac{d \phi_{\mathrm{bp}}}{d E_{\mathrm{g}}}=0.38$ for $\mathrm{Si}$ and $\frac{d \phi_{\mathrm{bn}}}{d E_{\mathrm{g}}}=0.7$ for InAs, which is because $E_{\mathrm{QC}}$ and $E_{\mathrm{QV}}$ depend on the electron and hole effective masses. In a simple picture where the NW is approximated to an infinite potential well, $E_{\mathrm{QC}(\mathrm{V})}=\frac{\hbar^{2} \pi^{2}}{m_{e(h)}^{*} W^{2}}$, where $W$ is the NW width, and $\phi_{\mathrm{bp}}^{\mathrm{NW}}$ and $\phi_{\mathrm{bn}}^{\mathrm{NW}}$ in the AI model can be written as,

$$
\phi_{\mathrm{bp}(\mathrm{n})}^{\mathrm{NW}}=\phi_{\mathrm{bp}(\mathrm{n})}^{\mathrm{Bulk}}+\frac{m_{e(h)}^{*}}{m_{e}^{*}+m_{h}^{*}} \Delta E_{\mathrm{g}},
$$

where the relation $\Delta E_{\mathrm{g}}=E_{\mathrm{QC}}+E_{\mathrm{QV}}$ is used. Note that $\frac{d \phi_{\mathrm{bp}}}{d E_{\mathrm{g}}}+\frac{d \phi_{\mathrm{bn}}}{d E_{\mathrm{g}}}=1$ for both the SI and AI model.

The second quantity we propose to measure in experiments involves $J_{0}$. As the extrapolated value of the cur-
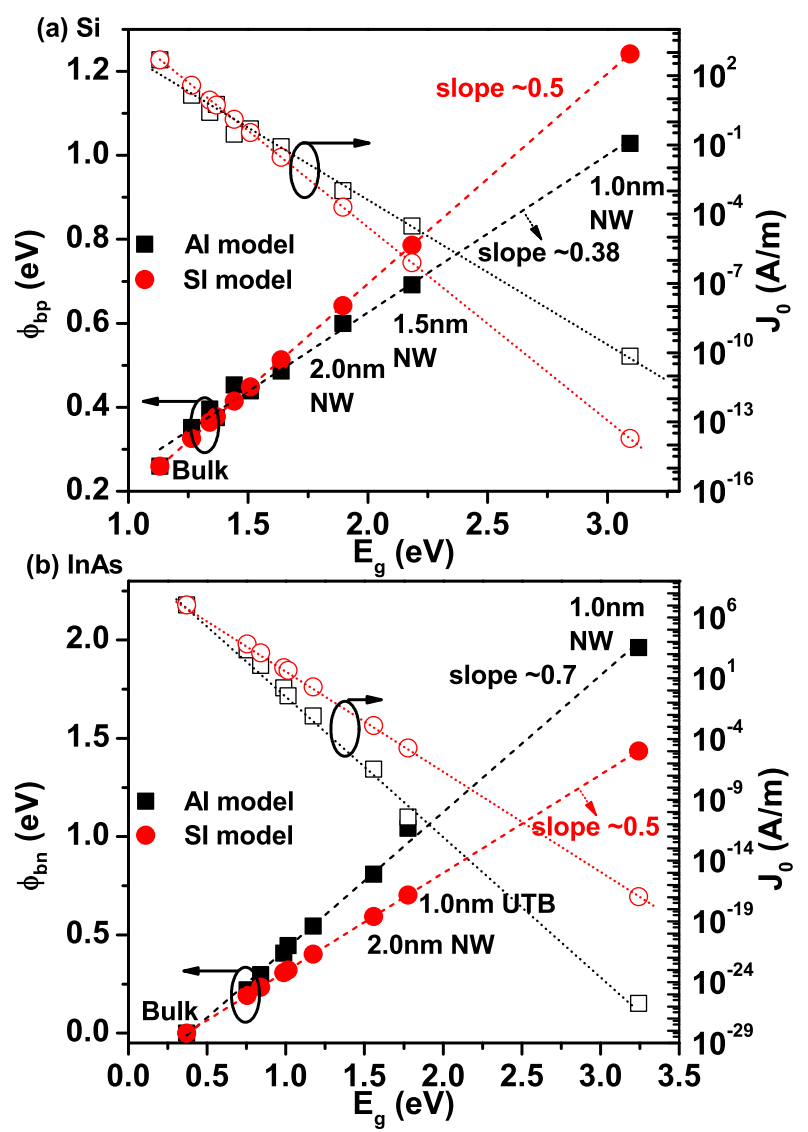

FIG. 6. The dependence of SBH and the saturation current density on the band gap for (a) Si and (b) InAs, respectively. $\phi_{\mathrm{bp}}^{\text {Bulk }}$ for $\mathrm{Si}$ and $\phi_{\mathrm{bn}}^{\text {Bulk }}$ for InAs are assumed to be 0.26 and $0.0 \mathrm{eV}$, respectively, based on the experimental results ${ }^{37,42-44,46}$. The SI model predicts $\frac{d \phi_{\mathrm{bp}(\mathrm{n})}}{d E_{\mathrm{g}}}=0.5$ or $\frac{d\left(\log _{10} J_{0}\right)}{d E_{\mathrm{g}}}=-8.4 \mathrm{dec} / \mathrm{eV}$. For the data generations in the figure, the $s p^{3} d^{5} s^{*}$ TB method is adopted with Boykin's parameters $^{47,48}$ and temperature is assumed to be $300 \mathrm{~K}$.

rent density at zero voltage in the SB diode, it is a parameter to assess the electrical properties of the metalsemiconductor interfaces. As shown in Fig. 6, $J_{0}$ decreases exponentially as $E_{\mathrm{g}}$ or $\phi_{\mathrm{bp}(\mathrm{n})}$ increases. Notice that the ratio between $J_{0}$ 's from the two models becomes exponentially large. In particular, it reaches as much as $3.7 \times 10^{3}$ and $6.2 \times 10^{8}$ for the Si and InAs NWs $(d=$ $1.0 \mathrm{~nm}$ ), respectively. The large difference in $J_{0}$ 's may signify the difference between the two models. In the SI model, $\frac{d\left(\log _{10} J_{0}\right)}{d E_{\mathrm{g}}}=-8.4 \mathrm{dec} / \mathrm{eV}$ for both Si and InAs, which readily follows from the same assumption applied to Eq. (3). In the AI model, on the other hand, we can analytically derive an equation for calculating $\frac{d\left(\log _{10} J_{0}\right)}{d E_{\mathrm{g}}}$ from Eq. (6) as:

$$
\frac{d\left(\log _{10} J_{0}\right)}{d E_{\mathrm{g}}}=-16.8(\mathrm{dec} / \mathrm{eV}) \times \frac{m_{e}^{*}}{m_{e}^{*}+m_{h}^{*}} .
$$

In conclusion, we have constructed a theoretical model 
for SBH of nanostructured silicide-silicon junctions, the SI model, which is capable of predicting SBH of nanostructures from SBH of the bulk junction. The SI model is the consequence of generalization of our DFT-GGA calculation results that $E_{\mathrm{FiF}}=E_{\mathrm{Fi}}^{\mathrm{Si}}-E_{\mathrm{F}}$ depends only on kinds of materials but not on the size of junctions. We also suggest two quantities, $\frac{d \phi_{\mathrm{bp}(\mathrm{n})}}{d E_{\mathrm{g}}}$ and $\frac{d\left(\log _{10} J_{0}\right)}{d E_{\mathrm{g}}}$, that can be used for experimental validation of our model. If the SI model serves for the prediction of $\phi_{\mathrm{b}}^{\mathrm{Nano}}$, they would be 0.5 (unitless) and $-8.4(\mathrm{dec} / \mathrm{eV})$, respectively, regardless of the kind of semiconductor materials. Experimental studies of SB for nano systems are extremely hard because of difficulty of systematic controlling and fabrication. We believe that our proposed model will improve the understanding of the SB formation mechanism, and guide experiments for improved SB-MOSFET developments.

${ }^{1}$ D.-Y. Jeon, S. pregl, S. J. Park, L. Baraban, G. Cuniberti, T. Mikolajick, and W. M. Weber, Nano Lett. 15, 4578 (2015).

${ }^{2}$ A. V. Penumatcha, R. B. Salazar, and J. Appenzeller, Nat. Commun. 6, 8948 (2015).

${ }^{3}$ J. M. Larson and J. P. Snyder, IEEE Trans. Electron Devices 53, 1048 (2006).

${ }^{4}$ S. Takagi, S.-H. Kim, M. Yokoyama, R. Zhang, N. Taoka, Y. Urabe, T. Yasuda, H. Yamada, O. Ichikawa, N. Fukuhara, M. Hata, and M. Takenaka, Solid-State Electronics 88, 2 (2013).

${ }^{5}$ W. Tang, S. A. Dayeh, S. T. Picraux, J. Y. Huang, and K.-N. Tu, Nano Lett. 12, 3979 (2012).

${ }^{6}$ S. Pregl, A. Heinzig, L. Baraban, G. Cuniberti, T. Mikolajick, and W. M. Weber, IEEE Trans. Nanotechnol. 15, 549 (2016).

${ }^{7}$ Y. Zhao, D. Candebat, C. Delker, Y. Zi, D. Janes, J. Appenzeller, and C. Yang, Nano Lett. 12, 5331 (2012).

${ }^{8}$ J. Guo and M. S. Lundstrom, IEEE Trans. Electron Devices 49, 1897 (2002).

${ }^{9}$ M. Shin, IEEE Trans. Electron Devices 55, 737 (2008).

${ }^{10}$ J. Lee and M. Shin, IEEE Electron Device Lett. 35, 726 (2014).

${ }^{11}$ E. Montes, K. Gkionis, I. Rungger, S. Sanvito, and U. Schwingenschlogl, Phys. Rev. B 88, 235411 (2013).

${ }^{12}$ U. Landman, R. N. Barnett, A. G. Scherbakov, and P. Avouris, Phys. Rev. Lett. 85, 1958 (2000).

${ }^{13}$ F. Leonard and A. A. Talin, Nat. Nanotechnol. 6, 773 (2011).

${ }^{14}$ G. P. Das, P. Blochl, O. K. Andersen, N. E. Christensen, and O. Gunnarsson, Phys. Rev. Lett. 63, 1168 (1989).

${ }^{15}$ H. Fujitani and S. Asano, Phys. Rev. B 42, 1696 (1990).

${ }^{16}$ H. Fujitani and S. Asano, Phys. Rev. B 50, 8681 (1994).

${ }^{17}$ M. K. Niranjan, S. Zollner, L. Kleinman, and A. A. Demkov, Phys. Rev. B 73, 195332 (2006).
${ }^{18}$ L. Geng, B. Magyari-Kope, and Y. Nishi, IEEE Electron Device Lett. 30, 963 (2009).

${ }^{19}$ Y. Nishi, T. Yamauchi, T. Marukame, A. Kinoshita, J. Koga, and K. Kato, Phys. Rev. B 84, 115323 (2011).

${ }^{20}$ Q. Gao and J. Guo, Appl. Phys. Lett. 99, 183110 (2011).

${ }^{21}$ Y. Jiao, A. Hellman, Y. Fang, S. Gao, and M. Kall, Scientific Reports 5, 11374 (2015).

${ }^{22}$ P. Srivastava, M. Shin, K.-R. Lee, H. Mizuseki, and S. Kim, AIP Advances 5, 087109 (2015).

${ }^{23}$ J. Kim, B. Lee, Y. Park, K. V. R. M. Murali, and F. Benistant, Proc. SISPAD , 226 (2015).

${ }^{24}$ X. Ma, Y. Dai, L. Yu, and B. Huang, Nanoscale 8, 1352 (2016). ${ }^{25}$ W. Schottky, Z. Physik 113, 367 (1939).

${ }^{26}$ N. F. Mott, Proc. R. Soc. (London) A 171, 27 (1939).

${ }^{27}$ A. M. Cowley and S. M. Sze, J. Appl. Phys. 36, 3212 (1965).

${ }^{28}$ R. T. Tung, Appl. Phys. Rev. 1, 011304 (2014).

${ }^{29}$ P. Ordejon, E. Artacho, and J. M. Soler, Phys. Rev. B 53, R10441 (1996).

${ }^{30}$ J. M. Soler, E. Artacho, J. D. Gale, A. Garcia, J. Junquera, P. Ordejon, and D. Sanchez-Portal, J. Phys.: Condens. Matter 14, 2745 (2002).

${ }^{31}$ J. P. Perdew, K. Burke, and M. Ernzerhof, Phys. Rev. Lett. 77, 3865 (1997).

${ }^{32}$ N. Troullier and J. L. Martins, Phys. Rev. B 43, 1993 (1991).

${ }^{33}$ M. Wittmer, Phys. Rev. B 42, 5249 (1990).

${ }^{34}$ H. Norde, J. de Sousa Pires, F. d'Heurle, F. Pesavento, S. Petersson, and P. A. Tove, Appl. Phys. Lett. 38, 865 (1981).

${ }^{35}$ M. O. Aboelfotoh, Phys. Rev. B 39, 5070 (1989).

${ }^{36}$ M. O. Aboelfotoh and K. N. Tu, Phys. Rev. B 34, 2311 (1986).

${ }^{37}$ V. W. L. Chin, J. W. V. Storey, and M. A. Green, Solid-State Electronics 32, 475 (1989).

${ }^{38}$ E. Alptekin and M. C. Ozturk, IEEE Electron Device Lett. 30, 1272 (2009).

${ }^{39}$ K. N. Tu, R. D. Thompson, and B. Y. Tsaur, Appl. Phys. Lett. 38, 626 (1981).

${ }^{40}$ T. Yamauchi, S. Zaima, K. Mizuno, H. Kitamura, Y. Koide, and Y. Yasuda, J. Appl. Phys. 69, 7050 (1991).

${ }^{41}$ R. T. Tung, A. F. J. Levi, J. P. Sullivan, and F. Schrey, Phys. Rev. Lett. 66, 72 (1991).

42 J. M. Andrews and J. C. Phillips, Phys. Rev. Lett. 35, 56 (1975).

${ }^{43}$ S. P. Murarka, J. Vac. Sci. Technol. 17, 775 (1980).

${ }^{44}$ G. Ottaviani, K. N. Tu, and J. W. Mayer, Phys. Rev. B 24, 3354 (1981).

${ }^{45}$ J. Tersoff, Surface Science 168, 275 (1986).

${ }^{46}$ H. H. Wieder, J. Vac. Sci. Technol. 21, 1915 (2003).

${ }^{47}$ T. B. Boykin, G. Klimeck, and F. Oyafuso, Phys. Rev. B 69, 115201 (2004).

${ }^{48}$ T. B. Boykin, G. Klimeck, R. C. Bowen, and F. Oyafuso, Phys. Rev. B 66, 125207 (2002). 